\title{
Nicht chirurgische Therapie der Dupuytrenkontraktur
}

\begin{abstract}
Die Injektion von Kollagenase aus Clostridium histolyticum ist eine hocheffektive Therapie der Dupuytrenkontraktur und könnte vielen Patienten die Operation ersparen.
\end{abstract}

— Die Dupuytrenkontraktur ist in fortgeschrittenen Stadien äußerst störend bei Alltagsverrichtungen. Die Standardtherapie der Dupuytrenkontraktur war bisher die offene Fasziotomie, die bei Kontrakturen der Gelenke von 30 Grad und mehr empfohlen wird.

Seit Kurzem steht mit der Kollagenase von Clostridium histolyticum eine Substanz zur Verfügung, die in der Lage ist, Kollagenablagerungen weitgehend aufzulösen. In einer randomisierten, placebokontrollierten und multizentrischen Doppelblindstudie wurden 308 Patienten mit Beugekontrakturen von 20 Grad

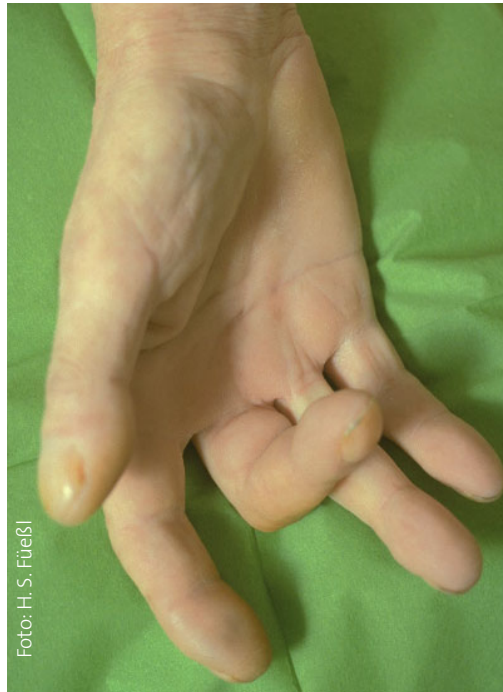

Dupuytrenkontraktur: Jetzt kann mit einer Spritze geholfen werden. kundären Endpunkte wie allgemeine klinische Verbesserung, mittlere Beweglichkeit in den betroffenen Gelenken im Vergleich zum Ausgangswert und Rezidivneigung war die Kollagenaseinjektion einer Placebobehandlung signifikant überlegen.

Der Bewegungsgrad unter Kollagenase bewegte sich zwischen 44 und 81 Grad, unter Placebo nur zwischen 45 und 49 Grad.

Als unerwünschte Begleiterscheikale Schwellungen, Schmerzen, Blutungen, Juckreiz und eine vorübergehende Lymphknotenvergrößerung im Bereich der abführenden Lymphwege auf. Insgesamt traten drei schwerwiegende Komplikationen auf, darunter zwei Sehnenabrisse und ein Fall eines komplexen regionalen Schmerzsyndroms. Veränderungen in und mehr im Bereich der Metakarpophalangeal- und der proximalen Interphalangealgelenke mit bis zu drei Injektionen von Kollagenase clostridium histolyticum bzw. Plcebo in den kontrakten Kollagenstrang behandelt.

Jeweils einen Tag nach der Injektion wurden die Gelenke passiv bewegt, um die Beugekontraktur zu lösen. Primärer Endpunkt der Studie war eine Rückbildung der Kontraktur bis in den Bereich 0-5 Grad bis zur vollen Streckung innerhalb von 30 Tagen.

Im Vergleich zu Placebo wurde mit der Kollagenaseinjektion bei einem signifikant höheren Anteil von Patienten der primäre Endpunkt erreicht $(6,8$ vs. $64,0 \%)$. Auch bezüglich der se-

Injectable collagenase clostridium histolyticum for dupuytren's contracture. New Engl. J. Med. 361 (2009) 10, 968-979 nungen traten lo-

\section{Online fortbilden und CME-Punkte sammeln}

\author{
Folgende Fortbildungen der $\mathbf{M} \mathbf{M W}$ \\ stehen Ihnen derzeit auf \\ www.CME-Punkt.de zur Verfügung \\ (Auswahl): \\ - Narkosevorbereitung beim Hausarzt \\ - Stomaversorgung \\ - Chronischer Husten im Kindesalter \\ - Der anämische Patient in der Praxis \\ - Akute Infekte der oberen Atemwege \\ - Impfungen für Reisende \\ - Moderne Asthmatherapie \\ - Konservative Therapie der stabilen KHK \\ - Akutes Koronarsyndrom \\ - Vorhofflimmern \\ - Tiefe Beinvenenthrombose
}

Die Teilnahme ist für Sie völlig kostenfrei. Melden Sie sich heute noch an.

\section{UND SO GEHT'S:}

$\checkmark$ Auf www.CME-Punkt.de gehen und sich einmal registrieren.

$\checkmark$ Dazu geben Sie persönliche Daten und Ihre Einheitliche Fortbildungsnummer (EFN) ein.

$\checkmark$ Nach der Registrierung stehen Ihnen sämtliche CME-Fortbildungen der Zeitschriften von Urban\&Vogel kostenfrei zur Verfügung. Für Hausärzte und Internisten besonders interessant: MMW, Der Hausarzt, Medizinische Klinik, GastroNews, Cardiovasc, InFo Diabetologie, PneumoNews.

$\checkmark$ Wenn Sie eine Fortbildungseinheit bestehen (bis zu 3 Punkte), können Sie sich Ihre Teilnahmebestätigung direkt als PDF herunterladen.

$\checkmark$ Wir empfehlen, diese Bescheinigungen gesammelt bei Ihrer Landesärztekammer einzureichen. 